\title{
A model of health care provider decision making about HPV vaccination in adolescent males
}

\author{
Andreia B. Alexander, MD, PhD, $\mathrm{MPH}^{1}$ \\ Candace Best, $\mathrm{PhD}^{1}$ \\ Nathan Stupiansky, $\mathrm{PhD}^{1}$ \\ Gregory D. Zimet, $\mathrm{PhD}^{1}$
}

\begin{abstract}
${ }^{1}$ Section of Adolescent Medicine, Department of Pediatrics, Indiana University School of Medicine
410 West 10th Street, HS 1001, Indianapolis, IN 46202; andreia.alexander@gmail.com; cabest@gru.edu; nstupian@iu.edu; gzimet@iu.edu
\end{abstract}

\section{Corresponding Author:}

Andreia B. Alexander, MD, PhD, MPH

Department of Emergency Medicine

Rutgers - New Jersey Medical School

30 Bergen Street

Newark, NJ 07101

andreia.alexander@gmail.com

(973) 972-9261 (Ph)

(973) 972-9268 (Fax)

This is the author's manuscript of the article published in final edited form as:

Alexander, A. B., Best, C., Stupiansky, N., \& Zimet, G. D. (2015). A model of health care provider decision making about HPV vaccination in adolescent males. Vaccine, 33(33), 4081-4086.

http://doi.org/10.1016/i.vaccine.2015.06.085 


\begin{abstract}
Introduction: In the U.S. HPV vaccination of adolescent males remains low, despite the recommendation for routine vaccination. Although research has highlighted that health care provider (HCP) recommendation is very influential in HPV vaccine uptake, research on this topic in the male population is lacking. Accordingly, we used a qualitative approach to identify HCP knowledge, attitudes, and behaviors regarding adolescent male HPV vaccination, one year after routine vaccination of adolescent males was recommended. Method: Twenty U.S. pediatric HCPs participated in 20-30 minute interviews about knowledge, attitudes, and practices regarding male HPV vaccination. Interviews were audiorecorded, transcribed and analyzed using inductive content analysis. Results: The providers had been in practice for $1-35$ years, $75 \%$ were female, and $75 \%$ were White. Opinions on HPV vaccination were shaped by knowledge/perception of the risks and benefits of vaccination. Although all providers frequently offered HPV vaccine to male patients, the strength and content of the offer varied greatly. Vaccination opinions determined what issues were emphasized in the vaccine offer (e.g., stressing herd immunity, discussing prevention of genital warts), while adolescent age influenced if and how they pitched their vaccine offer (e.g., HPV as an STI). Most providers agreed with the ACIP recommendations, however, several expressed that providers' pre-existing opinions might remain unchanged despite the recommendations. Consistent with the literature on determinants of HPV vaccination, providers believed that their own recommendation was a major factor in a family's decision to vaccinate. Barriers to vaccination included the "newness" and sexual nature of the vaccine, lack of insurance coverage and the vaccine not being mandated. Conclusions: Providers' opinions about, and approaches to offering, HPV vaccination to males were highly variable. Interventions designed to improve male HPV vaccination
\end{abstract}


should focus on helping providers to routinely recommend the vaccine to all of their eligible patients, both males and females.

Key Words: Human Papillomavirus, Health care provider, male, qualitative research, vaccine, adolescent

\section{Research Highlights}

- Perceptions of risks/benefits drive provider behaviors around male HPV vaccination

- Most providers recognize that their recommendation is important to male vaccination

- There is great variability in how providers offer the HPV vaccine to males

- Most providers agree with the ACIP recommendations for male vaccination

- Providers' pre-existing opinions remain unchanged despite ACIP recommendation 


\section{Introduction}

Human papillomavirus (HPV) has been identified as a necessary cause of genital warts, virtually all cervical cancers, many other anogenital cancers, and increasing numbers of cancers of the oropharynx(1). The quadrivalent HPV vaccine (HPV4) was licensed in the U.S. for 9 to 26 year-old males in 2009(2) and the Centers for Disease Control and Prevention's (CDC) Advisory Committee on Immunization Practices (ACIP) issued a routine recommendation for males in 2011(3). Although HPV vaccination has proven to be effective and safe, vaccination rates remain suboptimal in the U.S., especially for males. $(4,5)$.

Most published research on factors associated with HPV vaccine uptake in adolescents has focused on females(6-8). These studies have found that public insurance status, Hispanic race, lower family income, having received flu vaccine, increased perceived vaccine effectiveness, peer acceptance and anticipated inaction regret, were associated with increased vaccine uptake or uptake intent in males(6-10).

While some variation exists between the factors associated with male and female vaccine uptake, health care provider recommendation is a primary factor for HPV vaccination of both males and females $(11,12)$. However, many of the studies of males were conducted prior to implementation of the ACIP routine recommendation(13-15).

Although health care provider recommendation is very influential, research on this topic in adolescent males, post-routine ACIP recommendation, is lacking. This information is needed to better understand the nature of provider recommendation/non-recommendation and could be used to guide development of interventions aimed at improving provider vaccination of males. Accordingly, the 
purpose of this study is to identify health care provider knowledge, attitudes, and practices regarding adolescent male HPV vaccine recommendation, post-routine recommendation by the ACIP. As little is known about these factors, a qualitative approach was taken.

\section{Methods}

\subsection{Procedures}

Participants were a convenience sample of 20 pediatric physicians (general and adolescent) working in primary care clinics with relatively high vaccination rates serving primarily lower income families in a Midwestern city. Twenty-eight providers were contacted via email and asked to participate in a 20-30 minute interview about their opinions and practices related to HPV vaccination of males. Twenty (71.4\%) responded and interviews were conducted either in person, in a private room at the provider's clinic/research office or via telephone. Each participant was compensated with a \$20 gift card. Interviews were conducted after the 2011 ACIP recommendation for routine HPV vaccination of males. Written informed consent for this study was waived and the study was approved by the Institutional Review Board at the author's institution.

\subsection{Data Collection}

Prior to each interview, participants completed a demographic questionnaire. Semi-structured interviews were conducted by the primary author using an inductive approach based in grounded theory to avoid making assumptions, allowing for a model of decision making to emerge from the data. Interviews focused on practices regarding HPV vaccination for males. Topics included HPV vaccine knowledge, presentation of vaccine to patients, opinions about the need for male vaccination, risks, benefits, barriers, patient decision making, and school vaccination. 
Interviews were audio-recorded and transcribed. After each interview, the interviewer completed detailed field notes intended to document relevant information not captured on the audio-recording. The research team agreed that the data reached theoretical saturation upon completion of 20 interviews(16).

\subsection{Analysis}

Data were evaluated via inductive content analysis(17), which is utilized when little is known about a topic. The process includes open coding, creating categories, and abstraction of concepts, and tends to move from very specific to more general. The primary and secondary author read and openly coded four randomly chosen interviews identifying emerging issues, concepts, and themes surrounding the knowledge, opinions, and practices of health care providers regarding the HPV vaccine. The researchers then came together and discussed their individual codes and created a consensus list of preliminary codes. The researchers read through and coded the remaining 16 interviews separately and developed an initial model, which was readjusted throughout the process of coding until no new themes or changes to the model were detected. Disagreement between researchers was resolved through discussion.

\section{Results}

\subsection{Sample}

The 20 providers were mostly female $(n=15)$ and white $(n=15)$, and had been practicing pediatrics from 1 to 35 years (see Table 1 for more detailed information). Overall there were no notable differences in responses by gender or race.

\subsection{Model of Provider Recommendation}

After examining providers' knowledge, attitudes and practices around male HPV vaccination, a model of provider recommendation emerged (Figure 1). Below is an overview of the model, followed by 
a more detailed description of model components. We found that providers' knowledge and perception of the risks and benefits of the HPV vaccine for males influenced their opinions on male vaccination. Their opinions on male vaccination then determined the nature of their vaccine offer. While this was true for most providers, there were exceptions. For example, although one provider felt anal and penile cancer prevention were clear benefits of HPV vaccination in males, he felt uncomfortable discussing these cancers with parents and adolescent males. Additionally, he believed parents and adolescents would be equally uncomfortable discussing these cancers. Several other providers who acknowledged prevention of male cancers as a benefit of vaccination felt this was not an important benefit because the incidence of these cancers is low. As a result, these providers said that they did not discuss male cancers when offering HPV vaccine to males.

All providers agreed that their HPV vaccination offer influenced, at least partially, family vaccination decisions. For most providers, the age of the adolescent determined whether or not they would offer the vaccine and the components included in their vaccine offer. For example, a few providers stated that they never offer the vaccine to any males younger than age 11 , while other providers were willing to vaccinate at age 9. Furthermore, for some, the age of the male patient would determine whether or not they would discuss the sexually transmitted nature of the virus. Many providers felt the age of the adolescent influenced how families made the decision to vaccinate. Most providers stated that the older the adolescent, the more likely the adolescents' opinion was included in vaccination discussions and considered in vaccination initiation.

Most providers identified barriers to vaccination (e.g., "newness" of the vaccine, STI prevention) that influenced their offer and/or the decision of families to vaccinate. Similarly, many providers felt that logistical barriers (e.g., lack of insurance coverage, non-mandated school vaccination) strongly influenced families opting for non-vaccination for their sons. Interestingly, there was substantial variation in how providers felt that the ACIP's recommendation for routine male vaccination would 
affect vaccination rates. Most believed that the pre-existing opinions of individual providers would impact how they perceived the ACIP recommendation, which would then influence their vaccine offer (or non-offer). For example, if providers had a positive opinion about male vaccination, then they may add the ACIP recommendation to their offer in order to increase the "sell-ability" of the vaccine. Likewise, most interviewees believed that providers holding a negative opinion about male vaccination would not change their offer to include the latest recommendation and/or would not increase their frequency of male vaccine offers. A breakdown of model components and example quotes can be found in Tables 2 and 3, respectively.

\subsubsection{Benefits}

The most frequently reported benefits of HPV vaccination in males were prevention against genital warts $(n=14)$ and prevention of male to female HPV transmission $(n=14)$. While all of these providers felt that protection of females was an important aspect of male HPV vaccination, there was much more variation in beliefs about the importance of the prevention of genital warts, with some providers believing prevention of genital warts was important to personal health $(n=5)$ and for decreasing health care costs $(n=2)$, and others feeling that emphasis should only be placed on cancer prevention $(n=9)$. Other benefits listed by providers can be found in Table 2

Although penile, anal, and/or oral cancer prevention were considered benefits of male HPV vaccination $(n=9)$, these were mentioned by only a few providers (ranging from 5 for penile cancer to 2 for anal cancers). When asked why cancer prevention was not listed as a benefit for males, providers stated: 1) they did not know about the cancer indication $(n=3), 2)$ they knew that cancer prevention was indicated but did not know the specifics $(n=8)$, or 3 ) they did not feel the incidence of these cancers was high enough to be considered a benefit $(n=7)$. 


\subsubsection{Risks}

All of the providers agreed that the vaccine was safe and had a low side effects profile. The most frequently mentioned risks were increased pain compared to other vaccinations $(n=9)$ and syncope (mostly in females) ( $n=10)$. Other risks mentioned can be found in Table 2.

\subsubsection{Opinion}

Most providers viewed HPV vaccine as good, safe, and effective $(n=16)$. Although many believed males and females should be vaccinated equally $(n=9)$, several did not believe that vaccination was as important for males $(n=7)$. Some providers saw male vaccination as an important intervention primarily to establish herd immunity $(n=4)$.

\subsubsection{Offer}

Most providers stated they offered the vaccine to their male patients in conjunction with other routine adolescent vaccines $(n=18)$, but spent additional time explaining HPV vaccination for two main reasons: 1) its recent availability for males and 2) families' lack of awareness about male HPV vaccination. Oftentimes, providers would begin their offer by eliciting HPV vaccine information from the family $(n=7)$, then would describe the specifics about the vaccine. The most frequently mentioned components of the vaccine offer were that: 1$)$ the vaccine was used to prevent genital warts $(n=14)$ and cervical cancer ( $n=12), 2$ ) males should receive the vaccine in order to prevent future partners from being infected $(n=11), 3$ ) the vaccine was used to prevent an STI (n=8), and 4) the adolescent would need a series of three doses $(n=7)$. The majority of the providers concluded their offer with a personal recommendation that the adolescent male receive the vaccine $(n=11)$. However, only five $(25 \%)$ physicians in this study actually stated that they strongly recommend the vaccine to their male patients with another six (30\%) stating they recommend the vaccine, but not strongly. 
While many providers mentioned genital warts in their vaccine offer, some who worked with younger adolescents left it out completely due to its sexual nature $(n=5)$. In these instances, providers stressed cancer prevention in order to avoid this discussion. Conversely, most of the providers who spoke about genital warts excluded any discussion of anal cancers $(n=9)$. The most common reasons identified for this exclusion included provider discomfort and the perceived discomfort among families.

\subsubsection{Age}

Providers were evenly split about vaccinating young adolescents. Half were comfortable vaccinating 11-12 year old males ( $n=10)$, with some willing to start as young as age $9(n=3)$. The remaining providers were more reluctant to vaccinate young adolescent males $(n=10)$. Many of these providers noted the difficulty in explaining HPV vaccination to parents of younger adolescents because of the perception that parents are not ready to discuss sex or STIs.

\subsubsection{Barriers}

The most commonly reported barriers included the vaccine not being mandated for school attendance $(n=10)$, perceived lack of insurance coverage by private insurers $(n=9)$, anxiety around teen sexuality (8), males not coming back to complete the three dose series $(n=7)$, not all physicians offering/recommending the vaccine $(n=6)$, and the vaccine being too new $(n=4)$. Less commonly reported barriers can be found in Table 2.

\subsubsection{ACIP Recommendation}

Most providers agreed that the latest ACIP recommendation was beneficial $(n=13)$, with only a few providers questioning why the ACIP added a routine HPV vaccination for males ( $n=3)$. Most of the variation came with respect to the effect the recommendation would have on male vaccination and 
providers' knowledge about HPV vaccine and its recommendations for use. Most providers knew that the 2011 ACIP recommendation included routine HPV vaccination for males ( $n=11)$. However, some were unaware about this "new" recommendation (up to a year after the recommendation had been released) ( $n=2)$. Providers were knowledgeable that the vaccine should be given to adolescents, but were unclear on the specifics. For example, some knew that it could be given as young as age $9(n=5)$, but others did not. Likewise, most knew that it was routinely recommended for 11 and 12 year-olds $(n=18)$. Nevertheless, the majority did not know the upper age limit of the recommendation $(n=14)$. Most providers understood that the vaccine was indicated to prevent genital warts $(n=18)$, however, there was quite a bit of confusion around which male HPV-related cancers were included in the vaccination indications. Most providers became aware of the latest recommendation via newsletters and emails from the American Academy of Pediatrics and/or the $\operatorname{CDC}(n=8)$. Others were informed during office discussions with colleagues or formal meetings $(n=3)$.

\subsubsection{Decision}

There was very little variation regarding the decision process about male vaccination. Most providers believed that their recommendation was one of the most important factors in the family's decision $(n=15)$. This was evident in the reported percentage of males who accepted HPV vaccination. Providers who stated they recommended or strongly recommended the vaccine reported a 70-90\% vaccination uptake while providers who stated that they offered, but did not recommend the vaccine reported a vaccination rate of $25-50 \%$. Providers agreed that parents were the primary decision-makers around vaccination $(n=19)$. In the cases when adolescents had input into the decision, it was most likely to involve an older adolescent $(n=8)$.

\section{Discussion}


Although we were able to describe an overall model of provider offer, there was great variation within each of the components of the model, despite the fact that these providers were from the same or similar practice groups. This variability suggests that the decision to offer and recommend HPV vaccination is dependent on physician knowledge or lack of knowledge about, and opinions on, the risks and benefits associated with the vaccine.

One of the most heterogeneous findings was with regard to prevention of HPV-related cancers that affect males, with some providers having no/inaccurate knowledge of current indications and others having accurate knowledge. Providers who were more knowledgeable about HPV related cancers were also more likely to provide a strong recommendation for the vaccine. As provider recommendation is one of the strongest predictors of HPV vaccine uptake(11-14), future research should be conducted to determine effective interventions to increase physician knowledge about cancer-prevention indications for HPV vaccination.

A second area of heterogeneity was comfort with discussion about HPV vaccine as STI prevention. While some providers were personally uncomfortable discussing HPV outcomes such as anogenital warts, others believed that families would be uncomfortable with such a discussion, especially in younger adolescents. This set of findings is consistent with previous research, which shows that few pediatricians engage in meaningful discussions about sexual health with their adolescent patients(18). Not surprisingly, providers were more comfortable discussing sexual health issues with older adolescents ( $>15$ years old).

Overall, the providers considered physician recommendation to be the strongest determining factor in parent approval of vaccinating their children. However, despite an overall general positive opinion about male HPV vaccination, most providers did not provide strong recommendations for vaccination. It appears that most of the providers believed that male vaccination had a role in protecting female partners, but were less convinced of its direct benefits for their male patients, a finding consistent with 
research conducted prior to ACIP's routine recommendation (19). This finding may explain, at least in part, why U.S. male vaccination initiation rates since the ACIP routine recommendation have only reached $34.6 \%(4,20)$.

Finally, many of our respondents felt the latest ACIP recommendation would not have a major effect on provider recommendations; that a provider's pre-existing personal opinion of HPV vaccination would remain the principal determining factor. It is not clear to what extent this opinion is supported by data, but the slow progress in HPV vaccination rates of females and males, despite the strong routine ACIP recommendation, suggests that health care providers have not fully embraced the ACIP guidelines.

\subsection{Limitations}

While the qualitative design of this study allowed us to examine in-depth information about health care provider knowledge, attitudes, and practices around HPV vaccination, there are a number of limitations. Although a sample size of 20 interviews was adequate for this qualitative research study, it limits the generalizability of these findings, which may not be applicable to different groups of health care providers. Additionally, while this study provides a good starting point to understanding male HPV vaccination, the data collection sites were clinics with high adolescent vaccination rates and high proportions of patients on state funded insurance programs. Research conducted in clinics with lower vaccination rates as well as those with higher proportions of patients using private insurance will help elucidate the full range of issues surrounding providers and male HPV vaccination.

\section{Conclusion}

Overall, providers had a positive opinion about HPV vaccine and offered it to most of their male patients. There was substantial variability across providers in terms of knowledge about the benefits of HPV vaccination for males, comfort with discussing sexual issues with adolescents and their families, and 
whether or not the provider recommended the vaccine. The results of this study may help to inform the development and evaluation of interventions for physicians to increase vaccine uptake and may inform future, larger scale, research in diverse settings to gain a better understanding of physician knowledge, attitudes, and practices around HPV vaccination in general and of males in particular.

\section{CONFLICT OF INTEREST STATEMENT:}

Gregory Zimet and Nathan Stupiansky have been investigators on investigator-initiated research funded by Merck \& Co. In the last year, Gregory Zimet served as a consultant to Merck \& Co., Inc.

\section{ACKNOWLEDGMENTS}

This study was supported by Merck \& Co., Inc. through an investigator-initiated grant (MISP \#38094). Its contents are the sole responsibility of the authors and do not reflect the official view of Merck \& Co., Inc.

\section{References}

1. Center for Disease Control and Prevention. Human Papillomavirus 2014 [cited 2014 July 3]. Available from: http://www.cdc.gov/std/hpv/. 
2. US Food and Drug administration. October 16, 2009 Approval Letter - Gardasil 2009 [cited 2014 July 3]. Available from:

http://www.fda.gov/BiologicsBloodVaccines/Vaccines/ApprovedProducts/ucm186991.htm.

3. Centers for Disease Control and Prevention. Recommendations on the use of quadrivalent human papillomavirus vaccine in males - Advisory Committee on Immunization Practices (ACIP), 2011. 2011 December 23, 2011. Report No.: Contract No.: 50.

4. Elam-Evans LD, Yankey D, Jeyarajah J, Singleton JA, Curtis CR, MacNeil J, et al. National, regional, state, and selected local area vaccination coverage among adolescents aged 13-17 years-United States, 2013. MMWR. 2014;63:625-33.

5. Pierre-Victor D, Mukherjee S, Bahelah R, Madhivanan P. Human papillomavirus vaccine uptake among males 11-26 years in United States: Findings from the National Health and Nutrition Examination Survey, 2011-2012. Vaccine. 2014;32(49):6655-8.

6. Holman DM, Benard V, Roland KB, Watson M, Liddon N, Stokley S. Barriers to human papillomavirus vaccination among US adolescents: A systematic review of the literature. JAMA pediatrics. 2014;168(1):76-82.

7. Etter DJ, Zimet GD, Rickert VI. Human papillomavirus vaccine in adolescent women: a 2012 update. Current Opinion in Obstetrics and Gynecology. 2012;24(5):305-10.

8. Rambout L, Tashkandi M, Hopkins L, Tricco AC. Self-reported barriers and facilitators to preventive human papillomavirus vaccination among adolescent girls and young women: A systematic review. Preventive medicine. 2014;58:22-32.

9. Alexander AB, Stupiansky NW, Ott MA, Herbenick D, Reece M, Zimet GD. Parent-son decisionmaking about human papillomavirus vaccination: a qualitative analysis. BMC pediatrics. 2012;12(1):192. 10. Alexander AB, Stupiansky NW, Ott MA, Herbenick D, Reece M, Zimet GD. What parents and their adolescent sons suggest for male HPV vaccine messaging. Health Psychology. 2014;33(5):448. 
11. Lau $\mathrm{M}$, Lin $\mathrm{H}$, Flores $\mathrm{G}$. Factors associated with human papillomavirus vaccine-series initiation and healthcare provider recommendation in US adolescent females: 2007 National Survey of Children's Health. Vaccine. 2012;30(20):3112-8.

12. Rosenthal S, Weiss T, Zimet G, Ma L, Good M, Vichnin M. Predictors of HPV vaccine uptake among women aged 19-26: importance of a physician's recommendation. Vaccine. 2011;29(5):890-5.

13. Reiter PL, McRee A-L, Pepper JK, Gilkey MB, Galbraith KV, Brewer NT. Longitudinal predictors of human papillomavirus vaccination among a national sample of adolescent males. American journal of public health. 2013;103(8):1419-27.

14. Gilkey MB, Moss JL, McRee A-L, Brewer NT. Do correlates of HPV vaccine initiation differ between adolescent boys and girls? Vaccine. 2012;30(41):5928-34.

15. Malo TL, Giuliano AR, Kahn JA, Zimet GD, Lee J-H, Zhao X, et al. Physicians' Human Papillomavirus Vaccine Recommendations in the Context of Permissive Guidelines for Male Patients: A National Study. Cancer Epidemiology Biomarkers \& Prevention. 2014;23(10):2126-35.

16. Sandelowski M. Sample size in qualitative research. Research in nursing \& health. $1995 ; 18(2): 179-83$.

17. Elo S, Kyngäs H. The qualitative content analysis process. Journal of Advanced Nursing. 2008;62(1):107-15.

18. Alexander SC, Fortenberry JD, Pollak KI, Bravender T, Davis JK, Østbye T, et al. Sexuality talk during adolescent health maintenance visits. JAMA pediatrics. 2014;168(2):163-9.

19. Perkins RB, Clark JA. Providers' Attitudes Toward Human Papillomavirus Vaccination in Young Men Challenges for Implementation of 2011 Recommendations. American journal of men's health. 2012;6(4):320-3. 
20. Centers for Disease Control and Prevention. National and state vaccination coverage among adolescents aged 13-17 years- United States, 2012. 2013 August 30, 2013. Report No.: Contract No.:

34. 